\title{
Cation and light-responsive frameworks from non-canonical amphiphilic DNA nanostructures
}

\author{
Giacomo Fabrini, ${ }^{\dagger}$ Aisling Minard ${ }^{\dagger}$ Ryan A. Brady, $^{\ddagger}$ Marco Di Antonio, ${ }^{*} \dagger$ and \\ Lorenzo Di Michele*,†, \\ $\dagger$ Department of Chemistry, Imperial College London, London W12 0BZ, UK \\ $\ddagger$ Department of Chemistry, King's College London, London SE1 1DB, UK \\ IDepartment of Physics - Cavendish Laboratory, University of Cambridge, Cambridge CB3 \\ OHE, UK \\ E-mail: m.di-antonio@imperial.ac.uk; I.di-michele@imperial.ac.uk
}

\section{Abstract}

Thanks to its biocompatibility, versatility and programmable interactions, DNA has been proposed as a building block for functional, stimuliresponsive frameworks with applications in biosensing, tissue engineering and drug delivery. Of particular importance for in vivo applications is the possibility of making such nanomaterials responsive to physiological stimuli. Here we demonstrate how combining noncanonical DNA G-quadruplex (G4) structures with amphiphilic DNA constructs yields nanostructures, which we termed "Quad-Stars", capable of assembling into responsive hydrogel particles via a straightforward, enzyme-free, one-pot reaction. The embedded G4 structures allow one to trigger and control the assembly/disassembly in a reversible fashion by adding or removing $\mathrm{K}^{+}$ions. Furthermore, the frameworks can be rendered responsive to near-UV light through the addition of a porphyrin photosensitiser. The combined reversibility of assembly, responsiveness and cargo-loading capabilities of the hydrophobic moieties make Quad-Stars a promising candidate for biosensors and responsive drug delivery carriers.

\section{Main text}

Nucleic acids have proven to be valuable building materials for the designed self-assembly of $2 \mathrm{D}$ and 3D architectures, nanomachines and functional nanomaterials. ${ }^{1,2}$ This success is not surprising, as nucleic acids benefit from superior biocompatibility, versatility, and programmable interactions, while showing a consistent decrease in production price over time. ${ }^{3,4}$

DNA-based hydrogels, in particular, have shown promise in applications as varied as biosensing, ${ }^{5,6}$ drug delivery, ${ }^{7-9}$ synthetic bi$\operatorname{ology}^{10}$ and artificial cells. ${ }^{11}$ Key characteristics sought in DNA-based hydrogels include i) a fast, scalable and cost-effective production route, and ii) reversible stimuli-responsiveness, specifically the ability to alternate between two states, such as assembled (gel) or disassembled (sol), via two opposite stimuli.

Hybrid DNA-polymer hydrogels, usually constructed from polyacrylamide (PAAm) or polyethylene glycol (PEG) crosslinked via modified DNA side chains, have proven highly successful as biosensors and carriers, as they can respond to a wide range of stimuli, including thermal denaturation, ${ }^{5}$ strand displacement, ${ }^{12}$ small molecules opening DNA-aptamer bridges, ${ }^{13}$ and metal ions triggering cleavage through DNAzymes. ${ }^{14}$ Replacing DNA du- 
plexes with non-canonical i-motifs ${ }^{15}$ and Gquadruplexes $(\mathrm{G} 4 \mathrm{~s})^{16-18}$ further enables responsiveness to $\mathrm{pH}$ and cation concentration, respectively. A cooperative combination of both double-stranded DNA (dsDNA) linkers and $\mathrm{pH}$-responsive i-motifs has been used to produce shape-memory DNA hydrogels, with potential applications in tissue engineering and cellular scaffolding. ${ }^{19}$ However, the hybrid approach suffers from potentially toxic breakdown products, particularly in the case of acrylamidebased hydrogels, ${ }^{20}$ and it requires an additional production step to covalently link the DNA strands into the polymer matrix.

Conversely, all-DNA hydrogel frameworks can potentially feature a higher biocompatibility and biodegradability ${ }^{21}$ while simplifying the production route, and have been applied to controlled drug release ${ }^{22}$ and cell-free protein production. ${ }^{23,24}$ The inclusion of i-motifs ${ }^{25}$ and triplexes ${ }^{26}$ enables $\mathrm{pH}$-triggered, reversible sol/gel transitions, ${ }^{27}$ while DNA-aptamer bridges allow for hydrogel disassembly upon binding small molecule targets. ${ }^{28,29}$ Currently, though, pH-triggered assembly/disassembly is the only example of a reversible structural response driven in all-DNA hydrogels by a physiologically relevant stimulus, limiting the scope for biomedical applications of these materials.

Recently, we introduced a novel class of amphiphilic DNA nanostructures, dubbed CStars, ${ }^{30-32}$ that combine the versatility and programmability of DNA with the robust self-assembly of amphiphilic macromolecules. C-Stars consist of branched DNA junctions (nanostars) featuring a cholesterol moiety attached to the end of each arm, which enables the formation of crystalline or amorphous frameworks via the frustrated microphase-separation among the hydrophobic moieties and the hydrophilic DNA. C-Stars have proved very versatile, making it possible to tune the porosity of aggregates, reversibly entrap target proteins, and trigger aggregate disassembly $^{31}$ through toehold-mediated strand displacement. ${ }^{33,34}$ This response, however, requires non-biologically available single-stranded DNA as input, and is thus not directly applicable to in vivo scenarios.
Here, we expand upon the responsiveness of amphiphilic DNA nanostars by combining their robustness and versatility with a cationdependant DNA G4 motif. The resulting nanostructures, which we term Quad-Stars, form nano-porous frameworks responsive to biologically-relevant $\mathrm{K}^{+}$cations. The materials reversibly assemble in a simple, enzymefree, one pot reaction when exposed to $\mathrm{K}^{+}$ ions, the removal of which by chelation leads to disassembly. The amphiphilic character of the networks allows for loading of small hydrophobic cargo molecules, which can then be released upon disassembly. Besides $\mathrm{K}^{+}$chelation, the material can also be be disassembled upon addition of a G4-binding porphyrin photosensitiser and exposure to longwave UV light $(395-400 \mathrm{~nm})$, the combination of which, as previously described, disrupts the quadruplex through guanine photo-oxidation. ${ }^{35-39}$ This additional mechanism is irreversible but enables precise spatial control over the response. Owing to the combined assembly reversibility, multistimuli-responsiveness, and cargo-loading capabilities, we believe that Quad-Stars hold significant potential for applications such as smart biosensors and drug delivery carriers.

Quad-Stars are comprised of four identical duplex "arms" bearing a cholesterol endmodification which, under suitable conditions, can join via a tetra-molecular parallel G4 motif, as shown in Figure 1a-b. G-quadruplexes are non-canonical secondary structures that can form in guanine-rich nucleic-acid strands intra-molecularly, between two molecules or, as in Quad-Stars, between four distinct strands. ${ }^{40}$ Depending on the strand orientation, G4s can be parallel, antiparallel or hybrid. They comprise a series of stacked G-quartets interacting via Hoogsteen hydrogen bonding, as depicted in Figure 1a (top). Alkali metal ions, particularly $\mathrm{K}^{+}$, coordinate guanines, templating and stabilising G-quartets and their stacking into G4s. This grants G4s, and in turn Quad-Stars, a degree of responsiveness with respect to cationic environments.

As shown in Figure 1b, each Quad-Star arm assembles from three DNA strands, one bearing 

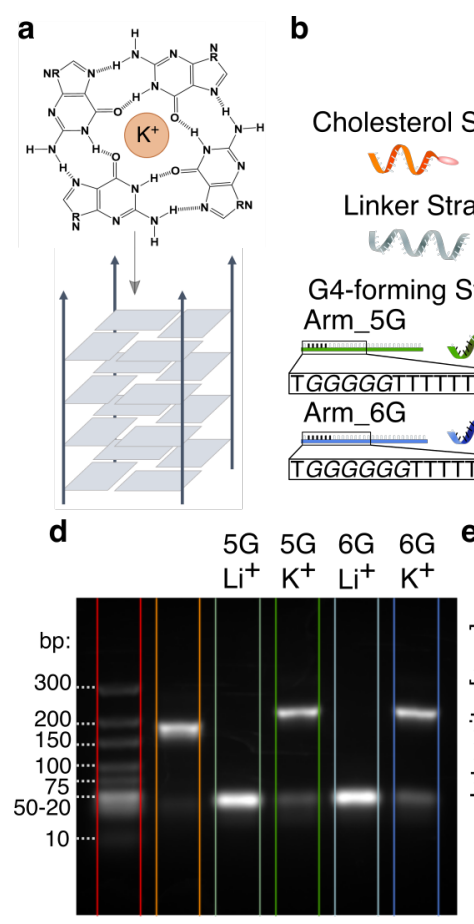

b

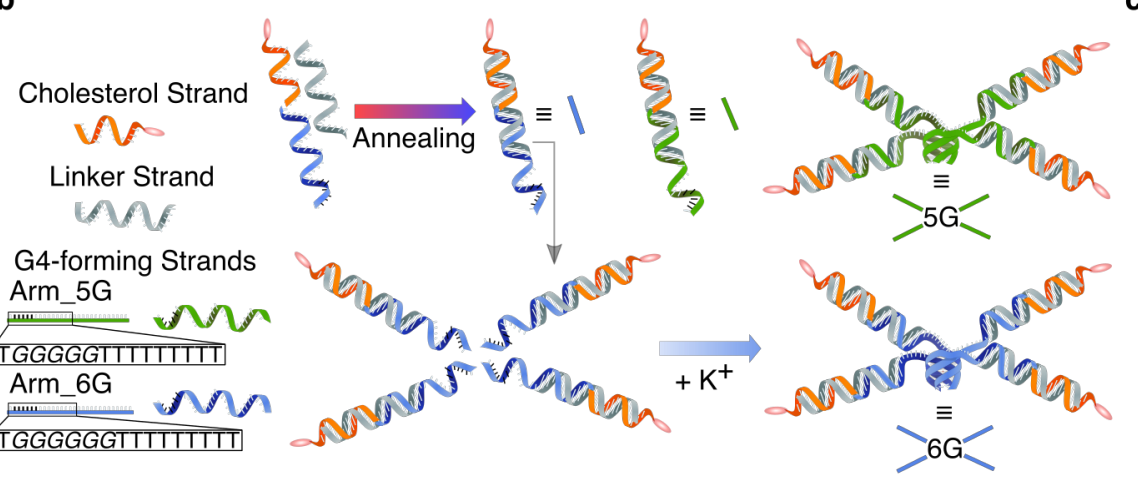

C

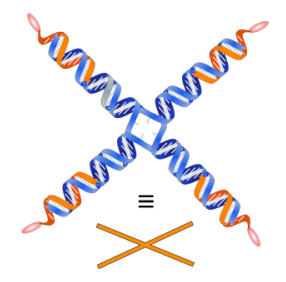

$f \frac{0}{9}$

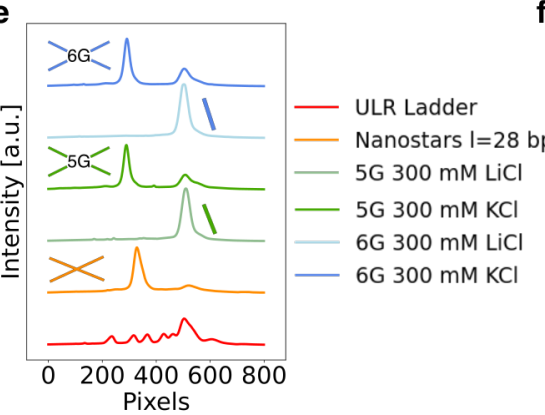

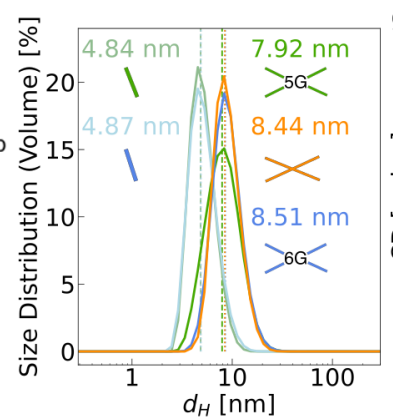

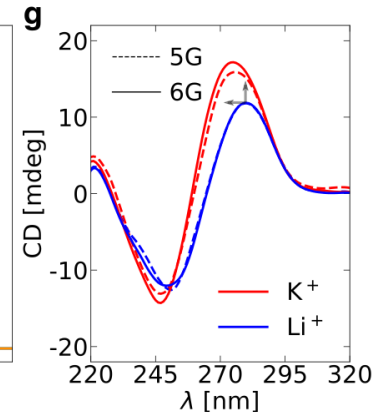

Figure 1: Design and characterisation of amphiphilic DNA Quad-Stars. (a) Schematic of a G-quartet (top) and a parallel tetramolecular G4 (bottom). (b) Quad-Stars assemble from four duplex arms, each made of three strands. During the annealing process, single-stranded DNA components hybridise into duplex arms, but G4 formation and assembly of the designed Quad-Star occurs only in the presence of $\mathrm{K}^{+}$ions. (c) Schematic of C-Stars ${ }^{30}$ with arm length $l=28$ base pairs (same as Quad-Stars). C-Stars and Quad-Stars share the same cholesterol-functionalised strands. (d) Agarose gel electrophoresis confirms that formation of individual, non-cholesterolised Quad-Stars is $\mathrm{K}^{+}$-dependent. Slower bands for both $5 \mathrm{G}$ (green) and 6G (blue) only appear when motifs are annealed in $\mathrm{KCl}$ $(300 \mathrm{mM})$, and their mobility is comparable to that of 4-arm nanostars with an arm length of 28 bp (orange). (e) Intensity profiles corresponding to the gel in (d). (f) Volume-based size distribution obtained via Dynamic Light Scattering (DLS) further substantiates the formation of Quad-Stars in $\mathrm{KCl}$, with a net shift from the size distribution in $\mathrm{LiCl}$. As expected from (d), the size of assembled motifs in $\mathrm{KCl}$ is comparable that of 4-arm nanostars in $300 \mathrm{mM}$ $\mathrm{NaCl}$. (g) Circular Dichroism (CD) proves that self-assembly relies on the formation of tetramolecular parallel G4s. Overlayed $\mathrm{CD}$ spectra in $\mathrm{LiCl}$ (blue) and $\mathrm{KCl}$ (red) clearly display the $\mathrm{K}^{+}$-induced shift (highlighted via arrows) in maximum and minimum towards $\approx 263$ and $240 \mathrm{~nm}$, respectively, as typical of parallel G4 structures. Samples for all reported measurements were prepared at [oligonucleotide] $=20 \mu \mathrm{M}$.

a cholesterol moiety, connected via a triethylene glycol (TEG) spacer, another acting as linker, and a third terminating with a 5 or 6 nucleotide (nt)-long guanine run flanked by thymines (T GGGGG T or T GGGGGG T, see zoomed inset in Figure 1b), a sequence capable of assembling into tetramolecular parallel G4s under physiological conditions. ${ }^{41}$ In the remainder of the text we refer to these designs as $5 \mathrm{G}$ and $6 \mathrm{G}$, respectively.

In the assembled arm, a poly-T spacer is present between the G-run and the duplex portion to allow for sufficient flexibility at the G4 site. Thymine-spacer lengths of 3, 5, 7 and $9 \mathrm{nu}-$ cleotides were tested, and minimal differences were observed in the Quad-Star self-assembly behaviour. In the remainder of the main text we report results for $9 \mathrm{~T}$ spacers, while analogous findings for different lengths are featured in SI.

Assembled Quad-Stars are topologically analogous to previously introduced C-Stars (Figure 1c), with the key difference that Quad-Star arms are held together by a stimuli-responsive G4-motif rather than an irreversible four-way junction. Similarly to C-Stars, ${ }^{30,31}$ we therefore expect Quad-Stars to self-assemble into nanoporous frameworks owing to the clustering of cholesterol moieties into micelle-like structures, coordinated and linked by the tetravalent DNA motifs. Unlike C-Stars, however, we expect 

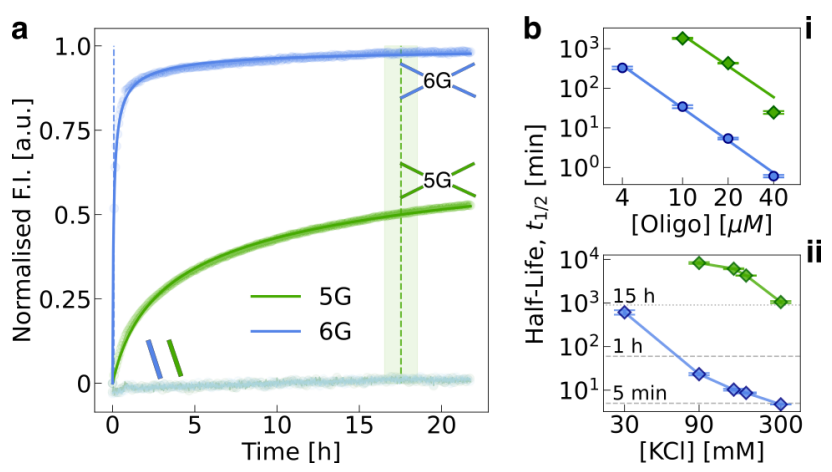

Figure 2: Kinetics of G4-dependent formation in soluble Quad-Stars via NMM fluorimetry. (a) Kinetic profiles of G4-folding at [Oligo] $=20 \mu \mathrm{M}$ (Oligo $=$ oligonucleotide) and $[\mathrm{KCl}]=300 \mathrm{mM}$ : as $\mathrm{G} 4 \mathrm{~s}$ form, NMM stacks on G-quartets and undergoes fluorescence emission enhancement. Normalised fluorimetry data are presented as mean (datapoints, one marker every 3 points shown) \pm standard deviation (shaded region) and fits (solid lines) to theoretical tetramolecular kinetics. ${ }^{41}$ Half-life values are reported as mean (vertical lines) \pm standard deviation (shaded intervals). Fainter coloured profiles at the bottom are negative controls (no $\mathrm{KCl}$ injection), reported as average datapoints only. (b) Half-life of formation Vs (i) [Oligo] at fixed $[\mathrm{KCl}]=300 \mathrm{mM}$ and (ii) $[\mathrm{KCl}]$ at fixed [Oligo $]=20 \mu \mathrm{M}$. (i) Data have been linearly fitted (solid lines) in log$\log$ scale according to power law $t_{1 / 2}=A \cdot[\text { Oligo }]^{1-n}$ to extract the reaction order from the fitting slope, resulting in $n=3.605 \pm 0.740$ for $5 \mathrm{G}\left(R^{2}=0.911\right)$ and $n=3.711 \pm 0.127\left(R^{2}=0.996\right)$ for $6 \mathrm{G}$. Both values closely match the expected stoichiometric value of 4 . Data in (b) is presented as mean (datapoints) \pm standard deviation (error bars). Negative controls in (a) are averages of independent duplicates. All other data has been averaged across (at least) triplicates. See Figure S6 (SI) for kinetic profiles for (b).

Quad-Star frameworks to respond to changes in ionic conditions. In particular, we expect Quad-Stars and Quad-Star networks to selfassemble at sufficiently high concentration of G4-promoting $\mathrm{K}^{+}$ions and, in turn, be destabilised by cationic species with a lower affinity for the G4 micro-environment, such as $\mathrm{Li}^{+}$or $\mathrm{Mg}^{2+}$ (Figure 1b).

To verify the ability of Quad-Star arms to assemble into the sought branched architectures at thermodynamic equilibrium and under suitable ionic conditions, samples of both the $5 \mathrm{G}$ and $6 \mathrm{G}$ designs were slowly annealed in TE buffer containing $300 \mathrm{mM} \mathrm{KCl}, 300 \mathrm{mM}$ $\mathrm{LiCl}$, or $2 \mathrm{mM} \mathrm{MgCl}_{2}$ (see Methods, SI). Net- work formation was here prevented by replacing the cholesterol-tagged strands with nonfunctionalised ones with identical sequence.

For both $5 \mathrm{G}$ and $6 \mathrm{G}$ samples annealed in $\mathrm{LiCl}$, Agarose Gel Electrophoresis (AGE) shows a single band corresponding to disjoined nanostar arms. When samples were instead annealed in $\mathrm{KCl}$, a second, slower band is present, which displays a mobility comparable to that of 4arm junctions (nanostars) with an arm length of $28 \mathrm{bp}$, and can thus be ascribed to correctly formed Quad-Stars (Figure 1d-e). Correct assembly was also confirmed by Dynamic Light Scattering (DLS) (Figure 1f), which clearly demonstrates the cation-dependent size shift for both $5 \mathrm{G}$ and $6 \mathrm{G}$ designs, with samples in $\mathrm{KCl}$ showing the same average hydrodynamic diameter as 4-arm nanostars with the same arm length. As shown by AGE (Figure S1) and DLS (Figure S2), samples annealed in $\mathrm{MgCl}_{2}$ exhibit partial Quad-Star assembly for the 6G design and no assembly for $5 \mathrm{G}$, hence pointing at a degree of quadruplex stabilisation intermediate between the lithium and potassium buffers.

Circular Dichroism (CD) spectra were used to confirm the presence, and determine the topology of the G4 motif (Figure 1g). Compared to those prepared in lithium, samples annealed in the $\mathrm{K}^{+}$-rich buffer present a clear shift in peak and valley positions towards 263 and $240 \mathrm{~nm}$ respectively, accompanied by an amplitude increase, indicating the formation of G4s with parallel strand orientation, as intended by design. Results for 5G and 6G Quad-Stars with different poly- $T$ spacer lengths can be found in Figures S3-S5 (SI). Structures employing G4forming strands with shorter G-runs (4G) were also tested and found incapable of linking the duplex arms in Quad-Star motif even in $\mathrm{KCl}$ buffers, producing results similar to those of negative controls where guanines are replaced by thymines (see Figures S3-S5, SI).

To explore the ability of Quad-Stars to isothermally respond to changes in ionic conditions, samples of non-cholesterolised QuadStars were initially annealed in $300 \mathrm{mM} \mathrm{LiCl}$ buffers, hence resulting in dispersed duplex arms, before triggering assembly via rapid in- 
jection of $\mathrm{KCl}$ (see Methods, SI). The assembly transient was then tracked by means of timeresolved fluorescence using the G4-sensitive probe N-methyl Mesoporphyrin IX (NMM), which displays a strong quantum yield increase upon preferential complexation with parallel G4s. ${ }^{42-44}$

The obtained time-dependant fluorescence intensity profiles display a monotonic signal increase after $\mathrm{KCl}$ injection, demonstrating G4 formation and isothermal assembly of QuadStars, and could be fitted to a simple model ${ }^{41}$ (see Methods, SI) to extract the assembly halflife, $t_{1 / 2}$, as exemplified in Figure 2a. The values obtained at the target conditions of $20 \mu \mathrm{M}$ oligonucleotide concentration (or equivalently [Quad-Star] $=5 \mu \mathrm{M}$ ) and $300 \mathrm{mM} \mathrm{KCl}$ clearly indicate that longer G-runs translate into faster assembly, with $t_{1 / 2} \sim 5$ min for $6 \mathrm{G}$ and $t_{1 / 2} \sim 15 \mathrm{~h}$ for $5 \mathrm{G}$.

In Figure 2b-(i) we explore the dependency of $t_{1 / 2}$ on Quad-Star concentration and, expectedly, we observe a steeply decreasing power-law trend that allows us to extract the reaction order of the assembly process as $n=3.605 \pm 0.740$ for $5 \mathrm{G}\left(R^{2}=0.911\right)$ and $n=3.711 \pm 0.127$ $\left(R^{2}=0.996\right)$ for $6 \mathrm{G}$, both values closely matching the expected stoichiometric value of 4 . Note that, owing to the prohibitively slow formation rate, assembly of the $5 \mathrm{G}$ motifs could not be tested at the lowest concentration used for $6 \mathrm{G}$. As shown in Figure 2b-(ii), Quad-Stars are found to fold significantly faster in the presence of higher potassium concentrations, with $t_{1 / 2}$ decreasing from $\sim 10 \mathrm{~h}$ to $\sim 5$ min when $\left[\mathrm{K}^{+}\right]$is increased from 30 to $300 \mathrm{mM}$ for the case of $6 \mathrm{G}$. A similarly strong dependence was found for $5 \mathrm{G}$.

Overall, the behaviours displayed in Figure $2 \mathrm{~b}$ demonstrate the ability of Quad-Stars to isothermally assemble in a cation and concentration-dependent fashion and, importantly, that assembly rates dramatically depend on both potassium and oligonucleotide concentrations.

Having verified that individual Quad-Star motifs correctly assemble from dsDNA arms in potassium-rich environments, we proceed to investigate the ability of cholesterolised QuadStars to form 3D percolating networks driven by hydrophobic interactions, as previously observed for C-Stars ${ }^{30-32}$ and sketched in Figure 3a-d.

As for C-Stars, we expected the duplex arms to form cholesterol-induced micelles even prior to Quad-Star formation. ${ }^{30}$ DLS in Figure S7 indeed confirms micelle formation in both $300 \mathrm{mM} \mathrm{LiCl}$ and $300 \mathrm{mM} \mathrm{KCl}$ (the latter for negative control samples where guanines are replaced by thymines to prevent G4 formation), while no micelles are observed in $2 \mathrm{mM} \mathrm{MgCl}_{2}$, probably owing to the much reduced ionic strength compared to the more concentrated monovalent-ion buffers. Consistently, while we observe no evidence of G4 formation in noncholesterolised Quad-Stars in the absence of $\mathrm{K}^{+}$ions, cholesterolised designs showed a moderate tendency to aggregation in $300 \mathrm{mM} \mathrm{LiCl}$. We suggest that the thermodynamic stabilisation of the quadruplex is due to colocalisation of the G4-forming domains within micelles. In contrast, cholesterolised arms annealed in $2 \mathrm{mM}$ $\mathrm{MgCl}_{2}$ show no aggregation, and were thus chosen as the starting condition for $\mathrm{K}^{+}$-triggered network-assembly experiments.

Upon addition of $\mathrm{K}^{+}$, Quad-Star networks are expected to assemble, as nanostar motifs, held together by G4 structures, bridge neighbouring micelles. As confirmed by time-resolved NMM fluorimetry in Figure 3b, indeed, the addition of $300 \mathrm{mM} \mathrm{KCl}$ rapidly triggers quadruplex formation. In comparison to non-cholesterolised designs for identical oligonucleotide and potassium concentrations, we observe a substantial boost in the assembly rate, particularly noticeable in 5G samples, which experience a 100fold rate increase (from $15 \mathrm{~h}$ to $10 \mathrm{~min}$ ). Direct comparison of $t_{1 / 2}$ between cholesterolised and non-cholesterolised designs is presented in Figure S9c. We propose that this rate increase is due to colocalisation of G4 motifs within cholesterol-induced micelles.

NMM fluorimetry assays in Figure 3b capture the isothermal folding of G4 motifs within cholesterolised Quad-Stars, rather than their aggregation into extended network phases. To verify the occurrence of the latter, and study 
a
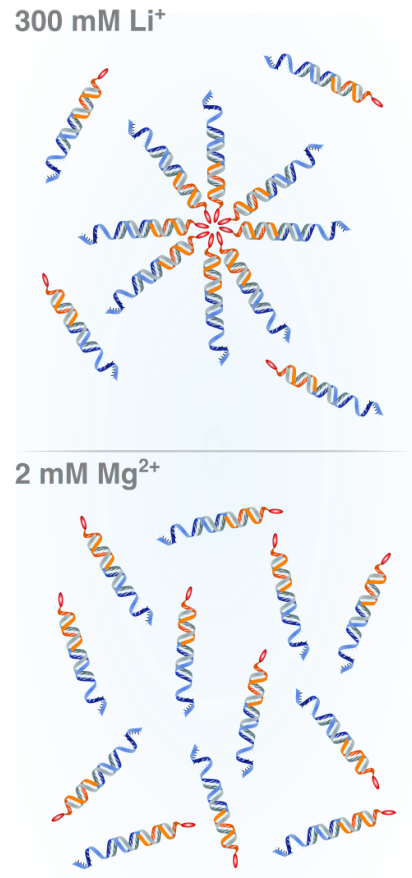

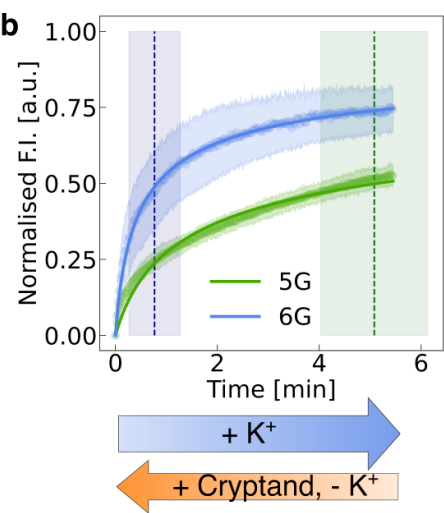

c

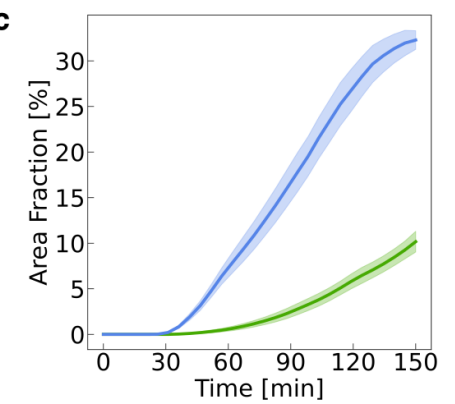

d



e

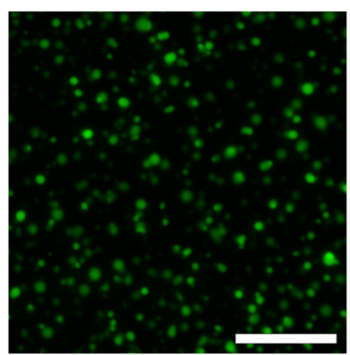

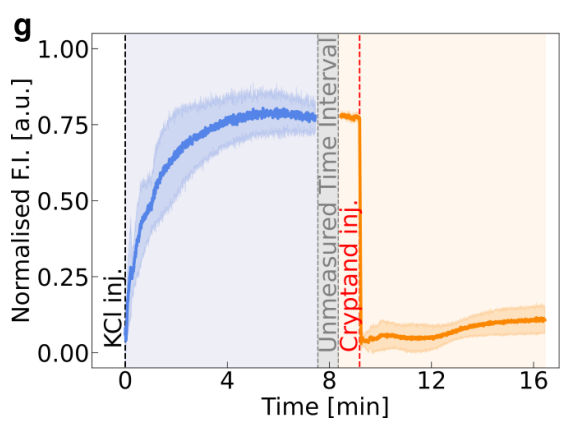

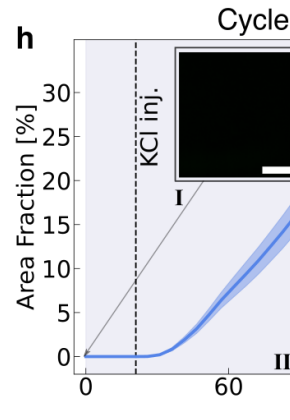

Cycle 1 Cycle 2

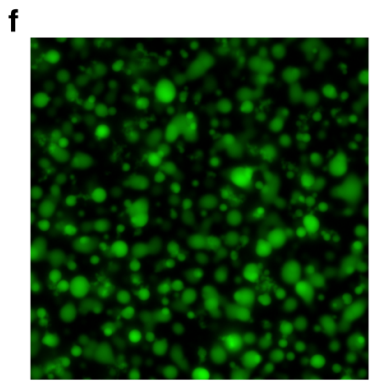

Cycle 3

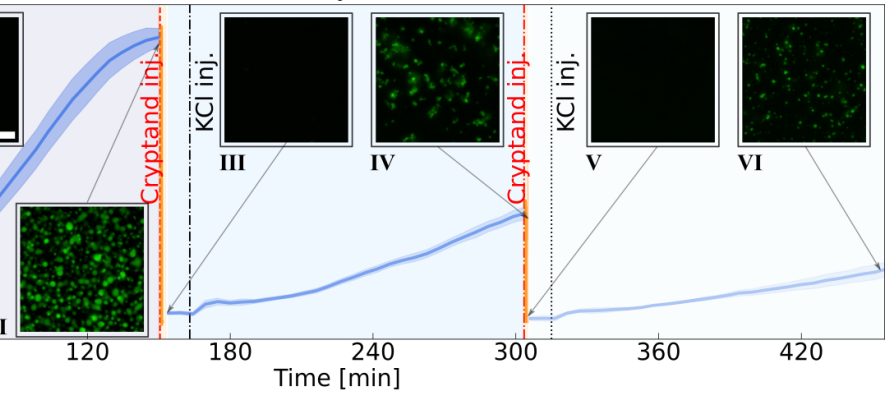

Figure 3: Reversible, isothermal assembly of Quad-Stars via NMM fluorimetry and epifluorescence microscopy. (a) Schematic representation of cholesterol-induced micelles and individual double-stranded DNA arms formed after annealing in $300 \mathrm{mM} \mathrm{LiCl}$ and $2 \mathrm{mM} \mathrm{MgCl}_{2}$, respectively (see Figure S7, SI). Presented data refers to samples annealed in $2 \mathrm{mM} \mathrm{MgCl}$. (b) Kinetic profiles of G4-folding in cholesterolised Quad-Stars via NMM fluorimetry. Normalised fluorimetry data are presented as mean (datapoints, one marker every 3 points shown) \pm standard deviation (shaded region) and fits (solid lines) to theoretical tetramolecular kinetics. ${ }^{41}$ Half-life values are reported as mean (vertical lines) \pm standard deviation (shaded intervals). See Figure S9 (SI) for examples of negative controls for 6G Quad-Stars. (c) Area fraction profiles extracted from epifluorescence microscopy timelapses depict the selfassembly kinetics of hydrogel aggregates. See control experiments in Figure S10 (SI). (d) Schematic representation of the 3D percolating network leading to hydrogel aggregate formation in cholesterolised Quad-Stars upon addition of $\mathrm{K}^{+}$ions. (e,f) Epifluorescence micrographs of 5G and 6G Quad-Star aggregates, respectively, formed after 2.5 h from potassium injection and imaged via an Alexa488 fluorophore linked to dsDNA arms. (g) Reversibility of assembly probed via NMM fluorimetry: addition of $\mathrm{K}^{+}$ions (black vertical line) leads to assembly, while their chelation by cryptand injection (red vertical line) leads to fast disassembly. Depicted sample is 6G. (h) Three consecutive assembly/disassembly cycles of $6 \mathrm{G}$ Quad-Stars demonstrate the reversibility of hydrogel aggregate formation. See Figure S11 (SI) for zoomed-in detail of disassembly profiles. Cropped micrographs in insets (I - VI) depict sample status at assembly stages indicated by arrows. Data in (c), (g) and (h) are shown as mean (solid lines) \pm standard deviation (shaded regions) of at least 3 repeats. Area fraction profiles in (c) and (h) were obtained via image segmentation in FIJI (see Methods, SI). Micrographs in panels and insets were background corrected via Rolling ball algorithm in FIJI (radius $=100 \mathrm{px}$ ). See Figure S12 (SI) for raw micrographs of (e)-(f). Scale bars are $50 \mu m$.

its kinetics, isothermal assembly was monitored via epifluorescence microscopy. For these experiments, aggregate visualisation was en- abled by an Alexa488 fluorophore chemically linked to one of the oligonucleotides (see Methods, SI) in order to prevent NMM-induced 
photo-destabilisation upon strong illumination. Oligonucleotide concentration was also reduced (from 20 to $5.6 \mu \mathrm{M}$ ) compared to the bulk fluorimetry assays, to enable the formation of discrete aggregates and facilitate image segmentation (see Methods, SI).

Assembly kinetics of network phases were monitored by determining the time-dependent area fraction of the microscopy field of view (FOV) occupied by aggregates (see Methods, SI). Figure $3 \mathrm{c}$ demonstrates a faster assembly rate for $6 \mathrm{G}$ compared to $5 \mathrm{G}$, leading to a higher area coverage after $150 \mathrm{~min}$ from $\mathrm{K}^{+}$injection, consistent with the trends in G4-folding determined by fluorimetry (Figure 3b). Micrographs in Figure 3e-f highlight the presence of round fluorescent aggregates after $\mathrm{K}^{+}$injection for both $5 \mathrm{G}$ and $6 \mathrm{G}$, with the latter displaying larger and more closely packed aggregates.

To disentangle the effect of increased ionic strength following $\mathrm{K}^{+}$injection from that of G4 stabilisation, in Figure S10 we monitor the response of samples annealed in $2 \mathrm{mM} \mathrm{MgCl}_{2}$ to the addition of $300 \mathrm{mM} \mathrm{LiCl}$. A degree of aggregation is observed, particularly with $6 \mathrm{G}$ designs, but this is significantly less pronounced than the response observed with $\mathrm{K}^{+}$, consistently with greater quadruplex-stabilisation ability of potassium and the evidence that $\mathrm{LiCl}$ only leads to limited aggregation at equilibrium (Figure S8). Consistent trends are observed in samples pre-annealed in $300 \mathrm{mM} \mathrm{LiCl}$ upon addition of further $300 \mathrm{mM} \mathrm{LiCl}$ or $300 \mathrm{mM} \mathrm{KCl}$ (Figure S10).

Potassium-stabilised G4 motifs are known to structurally weaken ${ }^{45}$ and rapidly unfold ${ }^{46-50}$ upon $\mathrm{K}^{+}$removal, which should lead to QuadStar and network disassembly. We assessed this reversibility route via NMM fluorimetry by injecting the high-affinity $\mathrm{K}^{+}$-binder ${ }^{46}$ and chelating agent [2.2.2] cryptand $^{50}$ after a potassium-induced assembly transient, while recording NMM fluorescent emission. Figure $3 \mathrm{~g}$ shows that the disassembly is almost instantaneous upon cryptand addition, as reflected by the sudden drop in fluorescence. This demonstrates the reversibility of G4 motif formation within cholesterolised Quad-Stars, which grants reversibility to the entire Quad-Star motif it- self.

To further demonstrate the reversibility of Quad-Star aggregation, we performed three subsequent rounds of isothermal assembly/disassembly by alternating $\mathrm{K}^{+}$and cryptand injections into the same $6 \mathrm{G}$ sample. Figure $3 \mathrm{~h}$ displays the associated timedependent area-fraction profile that demonstrates formation of visible aggregates after each potassium addition and an abrupt disassembly transition each time potassium ions are chelated. A slower assembly rate is observed for each subsequent potassium injection, likely due to the progressive dilution of the samples following multiple solution injections (see Methods, SI).

Therefore, we have demonstrated that QuadStars assemble into discrete hydrogel aggregates, with sizes ranging from a few to a few tens of microns, following a facile, enzyme-free isothermal reaction. Aggregate formation is reversible and responds to changes in cation concentration, exemplified by addition and chelation of the $\mathrm{K}^{+}$ions stabilising the inner $\mathrm{G} 4$ motifs.

The amphiphilic nature of Quad-Stars, combined with their reversibility and responsiveness, makes them promising candidates as delivery vectors for hydrophobic therapeutic molecules, which present a unique challenge in drug delivery due to their low solubility and poor bioavailability. ${ }^{51-54}$ Here we demonstrate the ability of Quad-Stars to encapsulate and release rhodamine $\mathrm{B}$, a model hydrophobic molecule which can be easily visualised via fluorescence microscopy.

Rhodamine B was added to samples prepared as for the isothermal assembly/disassembly experiments, prior to initiating hydrogel formation via $\mathrm{KCl}$ injection. Microscopy images in Figure 4a-b confirm that the formed QuadStar aggregates are indeed able to encapsulate hydrophobic rhodamine $\mathrm{B}$, as demonstrated by the higher fluorescence intensity detected within the aggregates as compared to the background: $27.2 \pm 4.7 \%$ for $5 \mathrm{G}$ and $34.7 \pm 3.4 \%$ for $6 \mathrm{G}$.

Disassembly of the cargo-loaded Quad-Star network through chelation of $\mathrm{K}^{+}$ions leads 
a

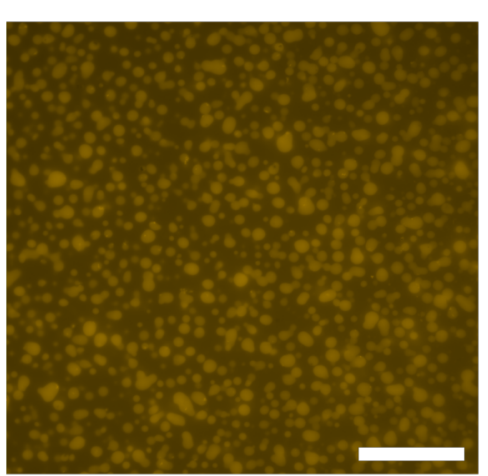

b

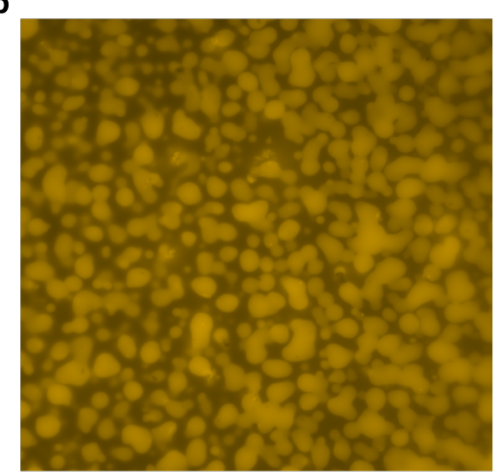

d

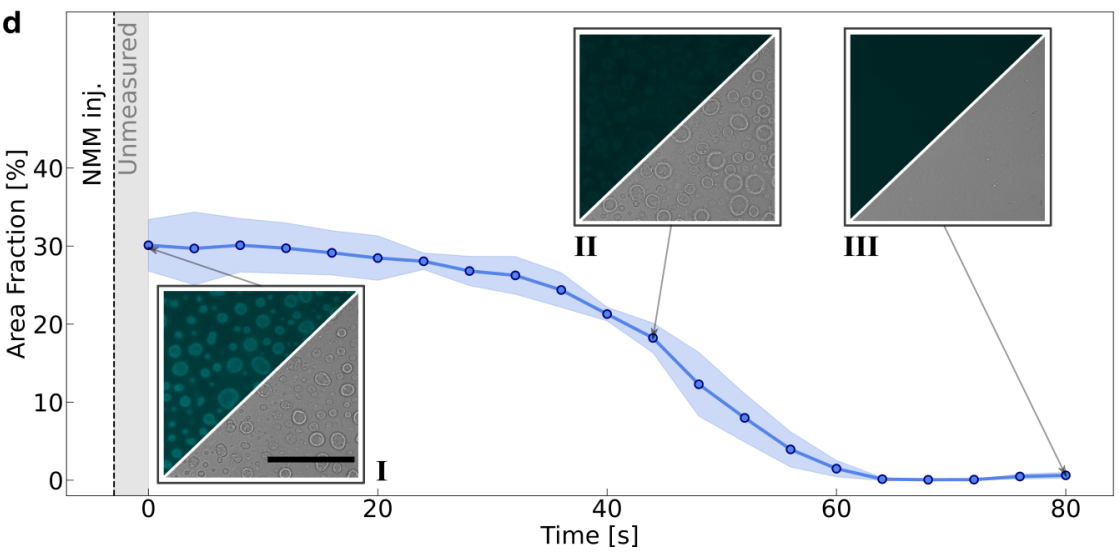

c

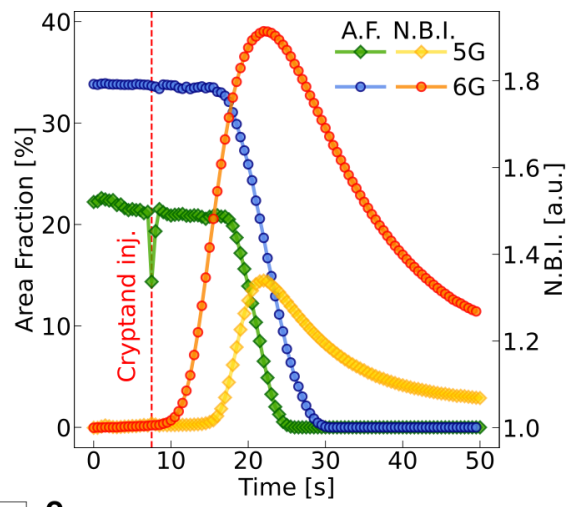

e

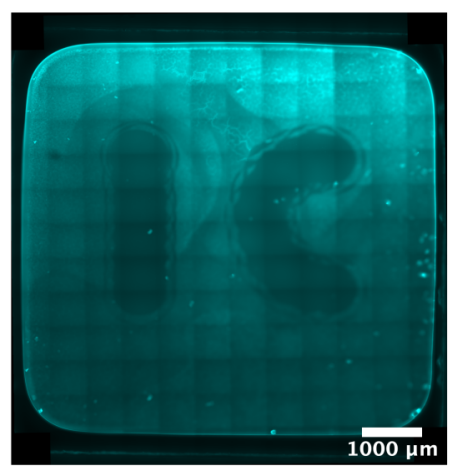

Figure 4: Uptake and release of model hydrophobic cargo rhodamine B. (a, b) Epifluorescence micrographs of $5 \mathrm{G}$ and 6G Quad-Star aggregates, respectively, at the end of a $3.5 \mathrm{~h}$ assembly run in the presence of rhodamine B. Hydrogel aggregates appear brighter than the background, proving preferential segregation of rhodamine B within the hydrophobic cholesterol pockets. (c) Representative profiles illustrating aggregate disassembly (A.F. = Area Fraction) upon cryptand injection (red vertical line), thereby causing the release of the rhodamine B cargo (N.B.I. $=$ Normalised Background Intensity). Profiles are extracted from single FOVs of representative samples, as release timescales strongly depend on the proximity of the injection to the FOV (see Figure S13, SI). (d) Light-induced disassembly via NMM photocleavage. NMM excitation $(395 \mathrm{~nm})$ leads to light-induced damage to G4 motifs and disassembly of hydrogel aggregates. Insets are composite images (bright-field and NMM fluorescence) corresponding to timesteps indicated by arrows. Data is presented as mean (circles) \pm standard deviation (shaded interval) of three FOVs within the same sample (6G Quad-Stars). (e) Photo-induced engraving in a sample well, reading "IC" (Imperial College), obtained by automatically exposing the pattern to $395 \mathrm{~nm}$ LED, thereby triggering location-selective NMMmediated photodisassembly. Scale bars are $50 \mu \mathrm{m}$, unless otherwise specified. Epifluorescence micrographs are unprocessed. Brigh-field insets have been contrast-enhanced for ease of visualisation. See Figure S14a-b (SI) for the raw images.

to release of rhodamine $B$ into solution. As demonstrated in Figure 4c, we follow the kinetics of Quad-Star network disassembly and cargo release simultaneously by monitoring the aggregate area fraction and background fluorescence intensity, respectively. Here, the cryptand solution was injected in the sample well far from the imaged FOV, hence a time delay is recorded between injection and disassembly, which is then observed as a sharp drop in the area fraction. Simultaneously, the background intensity displays a sharp increase, as rhodamine is released from the aggregates within the FOV and nearby locations in the sample, before slowly decreasing as the excess dye diffuses away from the site of release.

Besides enabling reversible control over aggregate formation depending on ionic conditions, the bridging G4 element of Quad-Stars opens up the possibility of triggering disassembly through a different, orthogonal stimulus, namely UV irradiation in the presence of NMM. While typically used as a fluorescent G4 probe (as in Figures 2-3b), NMM has indeed recently been reported to induce G4-photocleavage upon strong UV exposure. ${ }^{35}$ 
To test this response, we exposed assembled 6G Quad-Star hydrogels in the presence of NMM to longwave UV (395 nm LED), and then monitored the aggregates over time in both fluorescence (NMM emission at $670 \mathrm{~nm}$ ) and bright-field. As shown in Figure 4d, we observe rapid disassembly of Quad-Star aggregates following injection of NMM under UV exposure, with complete dissolution occurring within $90 \mathrm{~s}$. Aggregate disassembly is observed in both fluorescence and bright field channels (see Figure 4d, insets), proving that the observed effect is not due to NMM photobleaching, as also confirmed by fluorescence imaging of Alexa488-labelled samples (Figure S14c, SI). We observed this disassembly mechanism to be irreversible, in agreement with literature, which reports NMM-induced DNA photodamage and photocleavage selectively at G4 sites, most likely due to guanine photo-oxidation. ${ }^{35-39}$ A key advantage of light-induced disassembly is the possibility of activating a spatially localised response. To demonstrate this feature, we used the motorised microscope stage to expose a portion of a large $6 \mathrm{G}$ sample shaped as the letters "IC". Figure 4e shows selective disassembly of the Quad-Star aggregates in the exposed region and the emergence of the sought pattern. Note that the underlying square-lattice pattern in Figure $4 \mathrm{e}$ is an artefact of slightly uneven illumination and FOV stitching, rather than representing a physical characteristic of the sample.

In summary, Quad-Stars combine the robustness and versatility of amphiphilic DNA nanostars with the stimuli-responsiveness of noncanonical G4 motifs, thus allowing for controlled assembly and disassembly into hydrogel aggregates in response to biologically relevant $\mathrm{K}^{+}$cations.

We can tune the folding rates of individual motifs as well as the overall rate at which QuadStars aggregate into frameworks via multiple parameters, namely $\mathrm{K}^{+}$ion and oligonucleotide concentration, as well as the number of stacked G-quartets in the bridging G4s. The formed phases can naturally load small hydrophobic molecules, and later release them upon changes in the ionic environment. We believe they could be used, for instance, as drug delivery carriers exploiting cation concentration gradients existing among intra- and extra-cellular compartments. Moreover, in combination with a photosensitising porphyrin ligand, Quad-Star phases can be made to irreversibly disassemble upon localised UV exposure, unlocking a further control mechanism for cargo release.

Besides the direct applicability of these responsive nano-structured phases to encapsulation and controlled release of biomedically relevant cargoes, Quad-Star aggregates may find application as scaffolds for artificial, cell-like agents that many seek to construct in the context of bottom-up synthetic biology. ${ }^{55-58}$ Here, the responsive frameworks may act as membrane-less compartments, creating micro-environments which host active components whose action could couple with the responsiveness of the frameworks, hence achieving a unique synergy between structure and functionality. ${ }^{59-61}$

To this end, and more generally to further augment the range of factors regulating Quad-Star assembly/disassembly, we envisage the possibility of exploiting the helicase enzymes, either present in solution or tethered in the vicinity of the quadruplex-forming sites, or even potentially produced via cell-free protein expression systems, to selectively unfold G4s. ${ }^{62-65}$ The range of available responses could be further expanded thanks to the vast array of synthetic chemical species developed to tag, stabilise or de-stabilise G-quadruplexes for molecular biology assays or therapeutics. ${ }^{66}$

Finally, we note that while this contribution focused on the assembly/disassembly thermodynamics and kinetics of Quad-Star phases, the nano-structural properties of these materials remain to be discovered, particularly with respect to the ability of "regular" cholesterolised nanostars to form crystalline phases depending on design parameters such as flexibility and size. ${ }^{31,32}$ Quad-Stars could display a similar propensity towards the formation of ordered phases, but the added complexity of the geometrical and steric constraints imposed by the G4 motif could lead to the emergence of novel crystal geometries. 
Acknowledgement LDM acknowledges funding from a Royal Society University Research Fellowship (UF160152) and from the European Research Council (ERC) under the Horizon 2020 Research and Innovation Programme (ERC-STG No 851667 NANOCELL). GF acknowledges funding from the Department of Chemistry at Imperial College London. MDA and AM were supported by a BBSRC David Phillips Fellowship (BB/R011605/1).

\section{Supporting Information Avail- able}

Detailed experimental procedures and further supporting characterization data are available in the SI.

\section{References}

(1) Zhang, F.; Nangreave, J.; Liu, Y.; Yan, H. Structural DNA Nanotechnology: State of the Art and Future Perspective. J. Am Chem. Soc. 2014, 136, 11198-11211.

(2) Ramezani, H.; Dietz, H. Building machines with DNA molecules. Nat. Rev. Genet. 2020, 21, 5-26.

(3) Schmidt, T. L.; Beliveau, B. J.; Uca, Y. O.; Theilmann, M.; Da Cruz, F.; Wu, C. T.; Shih, W. M. Scalable amplification of strand subsets from chip-synthesized oligonucleotide libraries. Nat. Commun. 2015, 6, 1-7.

(4) Marchi, A. N.; Saaem, I.; Tian, J.; Labean, T. H. One-pot assembly of a hetero-dimeric DNA origami from chipderived staples and double-stranded scaffold. ACS Nano 2013, 7, 903-910.

(5) Liu, J. Oligonucleotide-functionalized hydrogels as stimuli responsive materials and biosensors. Soft Matter 2011, 7, 6757-6767.
(6) Xu, N.; Ma, N.; Yang, X.; Ling, G.; Yu, J.; Zhang, P. Preparation of intelligent DNA hydrogel and its applications in biosensing. Eur. Polym. J. 2020, 137, 109951.

(7) Sood, N.; Bhardwaj, A.; Mehta, S.; Mehta, A. Drug Delivery Stimuliresponsive hydrogels in drug delivery and tissue engineering Stimuli-responsive hydrogels in drug delivery and tissue engineering. Drug Delivery 2016, 23, 748-770.

(8) Nishida, Y.; Ohtsuki, S.; Araie, Y.; Umeki, Y.; Endo, M.; Emura, T.; Hidaka, K.; Sugiyama, H.; Takahashi, Y.; Takakura, Y.; Nishikawa, M. Selfassembling DNA hydrogel-based delivery of immunoinhibitory nucleic acids to immune cells. NBM 2016, 12, 123-130.

(9) Gačanin, J.; Synatschke, C. V.; Weil, T. Biomedical Applications of DNA-Based Hydrogels. Adv. Funct. Mater. 2020, 30, 1906253.

(10) Cui, J.; Wu, D.; Sun, Q.; Yang, X.; Wang, D.; Zhuang, M.; Zhang, Y.; Gan, M.; Luo, D. A PEGDA/DNA Hybrid Hydrogel for Cell-Free Protein Synthesis. Frontiers in Chemistry 2020, 8, 1-9.

(11) Samanta, A.; Sabatino, V.; Ward, T. R.; Walther, A. Functional and morphological adaptation in DNA protocells via signal processing prompted by artificial metalloenzymes. Nature Nanotechnology 2020, 15, 914-921.

(12) Lin, D. C.; Yurke, B.; Langrana, N. A. Mechanical Properties of a Reversible, DNA-Crosslinked Polyacrylamide Hydrogel. Journal of Biomechanical Engineering 2004, 126, 104-110.

(13) Zhu, Z.; Wu, C.; Liu, H.; Zou, Y.; Zhang, X.; Kang, H.; Yang, C. J.; Tan, W. An aptamer cross-linked hydrogel as a colorimetric platform for visual detection. Angewandte Chemie - International Edition 2010, 49, 1052-1056. 
(14) Lin, H.; Zou, Y.; Huang, Y.; Chen, J.; Zhang, W. Y.; Zhuang, Z.; Jenkins, G.; Yang, C. J. DNAzyme crosslinked hydrogel: a new platform for visual detection of metal ions w. Chemical Communications 2011, 47, 9312-9314.

(15) Tanaka, S.; Yukami, S.; Fukushima, K.; Wakabayashi, K.; Ohya, Y.; Kuzuya, A. Bulk pH-Responsive DNA Quadruplex Hydrogels Prepared by Liquid- Phase, Large-Scale DNA Synthesis. ACS Macro Letters 2018, 7, 295-299.

(16) Lu, C.-H.; Qi, X.-J.; Orbach, R.; Yang, H.H.; Mironi-Harpaz, I.; Seliktar, D.; Willner, I. Switchable Catalytic Acrylamide Hydrogels Cross-Linked by Hemin/ GQuadruplexes. Nano Letters 2013, 13, 1298-1302.

(17) Kahn, J. S.; Trifonov, A.; Cecconello, A.; Guo, W.; Fan, C.; Willner, I. Integration of Switchable DNA-Based Hydrogels with Surfaces by the Hybridization Chain Reaction. Nano Letters 2015, 15, 7773-7778.

(18) Tanaka, S.; Wakabayashi, K.; Fukushima, K.; Yukami, S.; Maezawa, R.; Takeda, Y.; Tatsumi, K.; Ohya, Y.; Kuzuya, A. Intelligent, Biodegradable, and Self-Healing Hydrogels Utilizing DNA Quadruplexes. Chemistry - An Asian Journal 2017, 12, 2388-2392.

(19) Guo, W.; Qi, X.-J.; Orbach, R.; Lu, C.H.; Freage, L.; Mironi-Harpaz, I.; Seliktar, D.; Yang, H.-H.; Willner, I. Reversible $\mathrm{Ag}+-$ crosslinked DNA hydrogels. Chemical Communications 2014, 50, 4065.

(20) Exon, J. H. A Review of the Toxicology of Acrylamide. J. Toxicol. Environ. Health, Part B 2006, 9, 397-412.

(21) Wang, D.; Hu, Y.; Liu, P.; Luo, D. Bioresponsive DNA Hydrogels: Beyond the Conventional Stimuli Responsiveness. Accounts of Chemical Research 2017, 50, 733-739.
(22) Um, S. H.; Lee, J. B.; Park, N.; Kwon, S. Y.; Umbach, C. C.; Luo, D. Enzyme-catalysed assembly of DNA hydrogel. Nature Materials 2006, 5, 797801.

(23) Park, N.; Um, S. H.; Funabashi, H.; $\mathrm{Xu}$, J.; Luo, D. A cell-free proteinproducing gel. Nature Materials 2009, 8, 432-437.

(24) Park, N.; Kahn, J. S.; Rice, E. J.; Hartman, M. R.; Funabashi, H.; Xu, J.; Um, S. H.; Luo, D. High-yield cell-free protein production from P-gel. Nature Protocols 2009, 4, 1759-1770.

(25) Cheng, E.; Xing, Y.; Chen, P.; Yang, Y.; Sun, Y.; Zhou, D.; Xu, L.; Fan, Q.; Liu, D. A pH-Triggered, Fast-Responding DNA Hydrogel. Angewandte Chemie - International Edition 2009, 48, 7660-7663.

(26) Lu, S.; Wang, S.; Zhao, J.; Sun, J.; Yang, X. A pH-controlled bidirectionally pure DNA hydrogel: Reversible self-assembly and fluorescence monitoring. Chemical Communications 2018, 54, 4621-4624.

(27) Guo, X.; Li, F.; Liu, C.; Zhu, Y.; Xiao, N.; $\mathrm{Gu}, \mathrm{Z}$; Luo, D.; Jiang, J.; Yang, D. Construction of Organelle-Like Architecture by Dynamic DNA Assembly in Living Cells. Angewandte Chemie - International Edition 2020, 59, 20651-20658.

(28) Simon, A. J.; Walls-Smith, L. T.; Plaxco, K. W. Exploiting the conformational-selection mechanism to control the response kinetics of a "smart" DNA hydrogel. Analyst 2018, $143,2538$.

(29) Oishi, M.; Nakatani, K. Dynamically Programmed Switchable DNA Hydrogels Based on a DNA Circuit Mechanism. Small 2019, 15, 1900490.

(30) Brady, R. A.; Brooks, N. J.; Cicuta, P.; Di Michele, L. Crystallization of Am- 
phiphilic DNA C-Stars. Nano Letters 2017, 17, 3276-3281.

(31) Brady, R. A.; Brooks, N. J.; Foderà, V.; Cicuta, P.; Di Michele, L. AmphiphilicDNA Platform for the Design of Crystalline Frameworks with Programmable Structure and Functionality. J. Am. Chem. Soc 2018, 140, 15384-15392.

(32) Brady, R. A.; Kaufhold, W. T.; Brooks, N. J.; Fodera, V.; Di Michele, L. Flexibility defines structure in crystals of amphiphilic DNA nanostars. J. Phys. Condens. Matter 2019, 31, 074003.

(33) Simmel, F. C.; Yurke, B.; Singh, H. R. Principles and Applications of Nucleic Acid Strand Displacement Reactions. Chemical Reviews 2019, 119, 6326-6369.

(34) Deluca, M.; Shi, Z.; Castro, C. E.; Arya, G. Dynamic DNA nanotechnology: Toward functional nanoscale devices. Nanoscale Horizons 2020, 5, 182-201.

(35) McBrayer, D.; Schoonover, M.; Long, K. J.; Escobedo, R.; Kerwin, S. M. N-Methylmesoporphyrin IX Exhibits G-Quadruplex-Specific Photocleavage Activity. ChemBioChem 2019, 20, 1924-1927.

(36) Morikawa, M.; Kino, K.; Oyoshi, T.; Suzuki, M.; Kobayashi, T.; Miyazawa, H. Product analysis of photooxidation in isolated quadruplex DNA; 8-oxo-7,8dihydroguanine and its oxidation product at 3-G are formed instead of 2,5-diamino4H-imidazol-4-one. RSC Advances 2013, 3, 25694-25697.

(37) Morikawa, M.; Kino, K.; Oyoshi, T.; Suzuki, M.; Kobayashi, T.; Miyazawa, H. Analysis of Guanine Oxidation Products in Double-Stranded DNA and Proposed Guanine Oxidation Pathways in SingleStranded, Double-Stranded or Quadruplex DNA. Biomolecules 2014, 4, 140159 .
(38) Kawauchi, K.; Urano, R.; Kinoshita, N.; Kuwamoto, S.; Torii, T.; Hashimoto, Y.; Taniguchi, S.; Tsuruta, M.; Miyoshi, D. Photosensitizers based on g-quadruplex ligand for cancer photodynamic therapy. Genes 2020, 11, 1-13.

(39) Cadet, J.; Richard Wagner, J. DNA base damage by reactive oxygen species, oxidizing agents, and UV radiation. Cold Spring Harb. Perspect. Biol. 2013, 5, a012559.

(40) Burge, S.; Parkinson, G. N.; Hazel, P.; Todd, A. K.; Neidle, S. Quadruplex DNA: Sequence, topology and structure. Nucleic Acids Res. 2006, 34, 5402-5415.

(41) Mergny, J. L.; De Cian, A.; Ghelab, A.; Saccà, B.; Lacroix, L. Kinetics of tetramolecular quadruplexes. Nucleic Acids Res. 2005, 33, 81-94.

(42) Li, Y.; Geyer, C. R.; Sen, D. Recognition of Anionic Porphyrins by DNA Aptamers. Biochemistry 1996, 35, 6911-6922.

(43) Ren, J.; Chaires, J. B. Sequence and structural selectivity of nucleic acid binding ligands. Biochemistry 1999, 38, 1606716075.

(44) Nicoludis, J. M.; Miller, S. T.; Jeffrey, P. D.; Barrett, S. P.; Rablen, P. R.; Lawton, T. J.; Yatsunyk, L. A. Optimized End-Stacking Provides Specificity of N-Methyl Mesoporphyrin IX for Human Telomeric G-Quadruplex DNA. J.Am.Chem.Soc. 2012, 134, 20446-20456.

(45) Bergues-Pupo, A. E.; AriasGonzalez, J. R.; Morón, M. C.; Fiasconaro, A.; Falo, F. Role of the central cations in the mechanical unfolding of DNA and RNA G-quadruplexes. Nucleic Acids Res. 2015, 43, 7638-7647.

(46) Aleman-Garcia, M. A.; Orbach, R.; Willner, I. Ion-responsive hemin-Gquadruplexes for switchable DNAzyme and enzyme functions. Chem. Eur. J. 2014, 20, 5619-5624. 
(47) Aizen, R.; Golub, E.; Trifonov, A.; Shimron, S.; Niazov-Elkan, A.; Willner, I. GQuadruplex-Stimulated Optical and Electrocatalytic DNA Switches. Small 2015, 11, 3654-3658.

(48) Olejko, L.; Cywiński, P. J.; Bald, I. An ion-controlled four-color fluorescent telomeric switch on DNA origami structures $\dagger$. Nanoscale 2016, 8, 10339-10347.

(49) Liu, X.; Zhang, J.; Fadeev, M.; Li, Z.; Wulf, V.; Tian, H.; Willner, I. Chemical and photochemical DNA "gears" reversibly control stiffness, shape-memory, self-healing and controlled release properties of polyacrylamide hydrogels $\dagger$. Chem. Sci. 2019, 10, 1008-1016.

(50) Chan, M. S.; Leung, H. M.; Wong, S. W.; Lin, Z.; Gao, Q.; Chang, T. J. H.; Lai, K. W. C.; Lo, P. K. Reversible reconfiguration of high-order DNA nanostructures by employing G-quartet toeholds as adhesive units. Nanoscale 2020, 12, 2464-2471.

(51) Gulyakin, I. D.; Oborotova, N. A.; Pechennikov, V. M. Solubilization of hydrophobic antitumor drugs (review). Pharm. Chem. J. 2014, 48, 209-213.

(52) Sareen, S.; Joseph, L.; Mathew, G. Improvement in solubility of poor watersoluble drugs by solid dispersion. Int. J. Pharm. Investig. 2012, 2, 12.

(53) Savjani, K. T.; Gajjar, A. K.; Savjani, J. K. Drug Solubility: Importance and Enhancement Techniques. ISRN Pharm. 2012, 2012, 1-10.

(54) Kalepu, S.; Nekkanti, V. Insoluble drug delivery strategies: Review of recent advances and business prospects. Acta Pharm. Sin. B 2015, 5, 442-453.

(55) Perrault, S. D.; Shih, W. M. Virusinspired membrane encapsulation of DNA nanostructures to achieve in vivo stability. ACS Nano 2014, 8, 5132-5140.
(56) Zhang, Z.; Yang, Y.; Pincet, F.; Llaguno, M. C.; Lin, C. Placing and shaping liposomes with reconfigurable DNA nanocages. Nat. Chem. 2017, 9, 653-659.

(57) Kurokawa, C.; Fujiwara, K.; Morita, M.; Kawamata, I.; Kawagishi, Y.; Sakai, A.; Murayama, Y.; Nomura, S. I. M.; Murata, S.; Takinoue, M.; Yanagisawa, M. DNA cytoskeleton for stabilizing artificial cells. PNAS 2017, 114, 7228-7233.

(58) Göpfrich, K.; Platzman, I.; Spatz, J. P. Mastering Complexity: Towards Bottomup Construction of Multifunctional Eukaryotic Synthetic Cells. Trends in Biotechnology 2018, 36, 938-951.

(59) Buddingh', B. C.; Van Hest, J. C. M. Artificial Cells: Synthetic Compartments with Life-like Functionality and Adaptivity. 2017,

(60) Jeong, S.; Nguyen, H. T.; Kim, C. H.; Ly, M. N.; Shin, K. Toward Artificial Cells: Novel Advances in Energy Conversion and Cellular Motility. Adv. Funct. Mater. 2020, 30, 1907182.

(61) Yewdall, N. A.; André, A. A.; Lu, T.; Spruijt, E. Coacervates as models of membraneless organelles. Curr. Opin. Colloid Interface Sci. 2021, 52, 101416.

(62) Sanders, C. M. Human Pif1 helicase is a G-quadruplex DNA-binding protein with G-quadruplex DNA-unwinding activity. Biochem. J. 2010, 430, 119-128.

(63) Wu, C. G.; Spies, M. G-quadruplex recognition and remodeling by the FANCJ helicase. Nucleic Acids Res. 2016, 44, 87428753 .

(64) Sauer, M.; Paeschke, K. G-quadruplex unwinding helicases and their function in vivo. Biochem. Soc. Trans 2017, 45, 1173-1182.

(65) Byrd, A. K.; Bell, M. R.; Raney, K. D. Pif1 helicase unfolding of G-quadruplex DNA 
is highly dependent on sequence and reaction conditions. J. Biol. Chem. 2018, 293, 17792-17802.

(66) Raguseo, F.; Chowdhury, S.; Minard, A.; Di Antonio, M. Chemical-biology approaches to probe DNA and RNA Gquadruplex structures in the genome. Chem. Commun. 2020, 56, 1317-1324. 